\title{
Mobile Data availability Similarity between Cooperative Caching and Replication
}

\author{
Sallam O Fageeri ${ }^{1}$, Rohiza Ahmad ${ }^{2}$, A.A.Mu'azu ${ }^{3}$ \\ 1, 2,3 (Department of Computer \& Information Science, Universiti Teknologi PETRONAS, Malaysia)
}

\begin{abstract}
Mobile technology presents many inherent problems that lead to poor network connectivity, low bandwidth. To overcome poor connectivity, Mobile clients are forced to operate in disconnected and partially connected modes. This paper review a designed method which led to support data availability using data replication and data caching in mobile environment to improving the availability of data and enhancing the performance of data access for users operates in mobile environments.
\end{abstract}

Keywords: Data Caching, Data Replication, Disconnect Operation, Mobile Computing

\section{INTRODUCTION}

Mobile computing led to gain increased attention to people and to their ability and efficiency of its services at any time and place, example of this query for billing, and weather forecast, as well as retrieval of information through the database of bank accounts is done through different working environment, in terms of the means of communication and the mediator through which is the transfer of information this is done through a special application that supports mobile computing, which are among several parties, leading to some concerns in handling queries [1] .

Data Management in mobile computing poses great challenges to the researchers. One of the important challenges is to provide a data caching or data replication solution, suitable for various applications in mobile environment. One of the important challenges for the researchers is how to solve data management, one of them is how to increase availability by getting a good way for data caching and data replication [2]

Caching is a very important technique to increase and improve the performance of wireless networks. Cooperative caching has give significant attention to the context of wireless sensor networks and ad hoc networks, it is beneficial to cache frequently accessed data items not only to reduce the average query latency, but also to save wireless bandwidth and extend the battery life [3] .

The concept of replication on the mechanism of data replication, and then keep them in multiple places, separate from the primary source, and can include all the database, or part thereof, and designed some of the methods that allow replication and through dealing with the data by users duplicate copy of the basic version independently to improve the continuity of work, where this is achieved when there is no availability of the source [4]

\section{challenges faced in mobile technology}

- $\quad$ Limited Resources: The CPU power and storage of mobile devices is continuously increasing; however, still they are far behind non-mobile systems such as servers on the Internet. Due to the size of the database, limited CPU power, storage capacity mobile device need to perform simple operations on local data available in cache. Limited storage capacity also makes it difficult to cache entire databases to a mobile device.

- $\quad$ Power consumption: The most prominent limitation of mobile device is power; these devices rely entirely on battery power. Combined with the compact size of many mobile devices, this often means unusually expensive batteries must be used to obtain the necessary battery lives.

- Disconnection: Weather, terrain, and the range from the nearest signal point can all interfere with signal reception. Reception in tunnels, some buildings, and rural areas is still poor. Interaction between a mobile device and a database is directly affected by the device's network connectivity.

- Insufficient bandwidth: Mobile access is generally slower than direct cable connections. Using technologies such as GPRS and EDGE, and more recently $3 \mathrm{G}$ networks bandwidth has been increased but still less compared to the wired network. Asymmetry problem is faced, where bandwidth in the downstream direction is often much greater than bandwidth in the upstream direction [5][6][7] .

- Mobile units are powered by limited capacity rechargeable batteries. Without continuous power supply, their life duration is finite (in the order of a few hours for laptop computers and a bit longer for smart 
phones and PDAs), whereas desktop computers are continuously powered and have virtually infinite power. In fact, limited battery life is an important reason for mobile devices being fitted with resources that are not very powerful but consume less energy. It is, therefore, very important to develop methods that utilize battery efficiently.

- Limited coverage of mobile networks: While traditional cellular networks provide all around coverage, high speed data connections are currently limited to areas with high user density. Besides, obstacles which will not allow radio signals to be reached, such as buildings and mountains along with signal loss and various types of noise. This will always leave some mobile users without network connectivity for various durations. Therefore, methods used for operating in mobile environments cannot assume continuous network coverage. Strategies to overcome temporary disconnections need to be considered.

High cost of accessing mobile networks: The cost of data access in mobile networks is far greater than wired networks. With the increasing of extensive-data in mobile applications, such as visual multimedia delivery systems, the demand for wireless bandwidth can be expected to be insufficient in the future. This demand cannot be easily serviced because of inadequate spectrum availability. Therefore, the monetary cost of wireless networks would not be less than or equal to wired networks. Further, intangible costs such as processing requirements and activating the wireless communication interface are important considerations as they consume a finite power source and directly affect the duration for which the mobile device can be used without recharging. The user perception of intangible costs is very important as it directly affects their usability. Therefore, methods for operating in mobile environments need to be extremely sensitive to monetary as well as intangible costs [6] [7][8][9][3][10].

\section{LITERETURE REVIEW}

Many research efforts had been done in the area of mobile data management's exactly in mobile data caching and mobile data replication here we can introduce some of proposed methods .

\section{Cooperative Data caching}

Three caching schemes known as: Cache-Path, Cache-Data, and Hybrid Cache. The main idea for these three concepts is to analyze passing requests and cache the data or the address of the node at its stored place. Later, if the same data requested passes through the node, it can provide the data itself or redirect the request to its specific location. Cache-Path Figure 1 saves space by storing locations where data should be stored, while Cache Data saves the time by storing the data. The third scheme, Hybrid-Cache, is a combine solution where queries are cache by path or by data, depending on what is optimum [8]

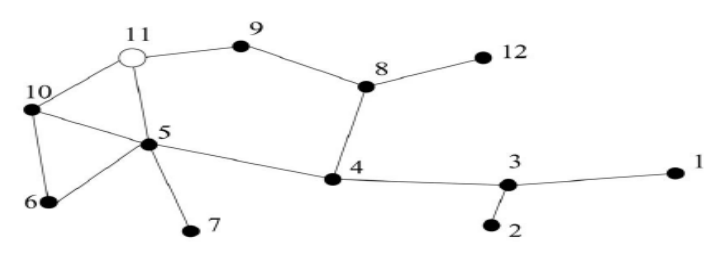

Fig 1 Caching path in MANET [8]

A caching algorithm is suggested to minimize the delay when acquiring data. In order to retrieve the data as quickly as possible, the query is issued as a broadcast to the entire network figure 2 . All nodes that have this data are supposed to send an acknowledgment back to the source of the broadcast. The requesting node $(\mathrm{RN})$ will then issue a request for the data (unicast) to the first acknowledging node it hears from. However, the scheme is inefficient in terms of bandwidth usage because of the broadcasts, which, if frequent, will largely decrease the throughput of the system due to flooding the network [9].

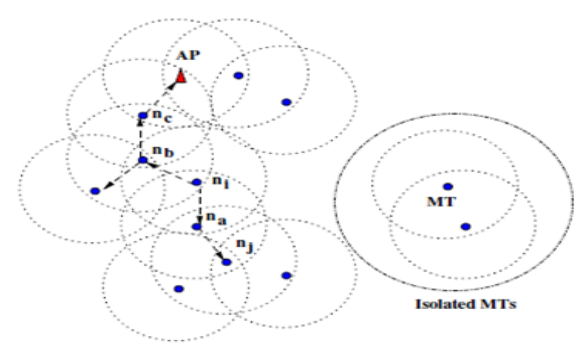

Fig2 Broadcasting for entire network [9] 
COOP is protocol based on maintaining history details (source and position of "passing" data) in nodes of a MANET figure 3 , and defining cooperation zones. Each node maintains a table where the received requests are recorded. Initially, a node checks its table after its local misses and before flooding a request. If matching entries are found, the node compares its distances to these matching caches and the original data source, and selects the closest node to redirect the request. In case of table miss, the requester node is using an adaptive z-hop flooding technique. If adaptive flooding fails, the request is sent to data source for response [10].

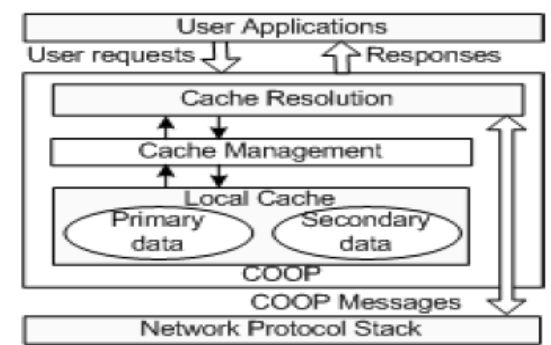

Fig3 COOP in nodes of a MANET [10].

Simple Cache which is known as on-demand data access applications, the traditional way of resolving a data request is to check the local cache first and send the request to the data server after local cache misses. This scheme is referred to as Simple Cache, This scheme works well as long as the connection to the data server is reliable and not too expensive; otherwise, it results in failed data requests or request timeouts[11][12]

Data caching scheme which is capable of intelligent pre-fetching for mobile ad hoc networks (MANETs) figure4. pre-fetching works as a integrated part of data caching, the mechanism is try to sense the future needs of mobile nodes (MNs), once it is established regarding the requirement of a particular data item, data is fetched and saved in the cache before it is required, but pre-fetching consumes resources such as computation power and energy[13].

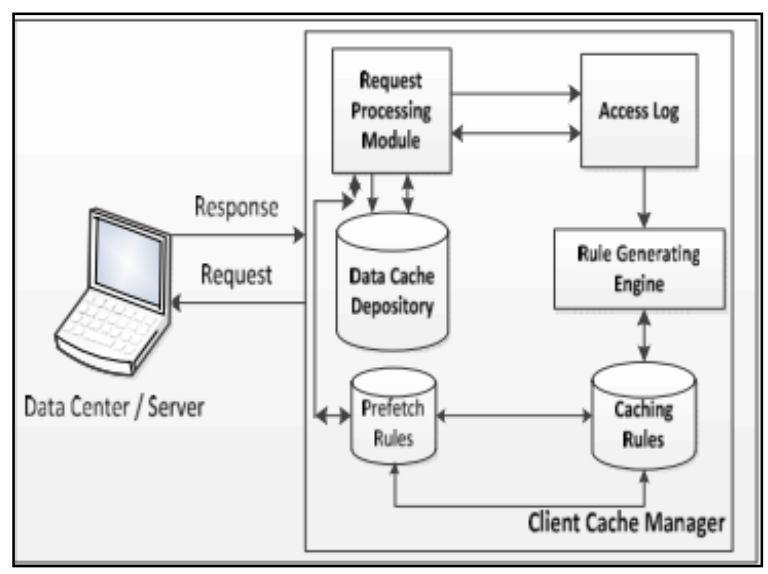

Fig 4 prefetch in MANETs [13].

\section{Data replication}

Applied algorithm called (ERDA) which is based on remote data access (MS RDA) the address and the organization to bridge the gap existing in the algorithm and the reform of the structure of databases and replication in the cell so as to avoid overlapping groups of data on the relationship between the server and the client through to find a way appropriate division of these relations, an example of this geographical location, and through which can reduce the number of replicas and avoid conflicts [14].

A Meta dynamic update protocol in replicated database, allows adjustment scenarios in which they can change the consistency and coherence, according to the requirements of the applications needs. And showed the extent of basic services for distributed systems, and reliable messaging and opinions can help to get this kind of functionality. He claimed that can be integrated into such a protocol definition and the commercial database programming interfaces by means of the orientation and, therefore, is currently a prototype implementation of this Protocol, the current definition. It is important to achieve deeper relationships between the views and problems update protocol, as it had done with the consensus and agreement problems other features offered are an initial step in the definition of the update problem for systems of transactions replicated, further research that show and can be done by the "isolation" between the copies and control protocols, protocol executions 
metadata can be defined as characteristics of the strongest and weakest on the basis of "dynamic update protocol levels of isolation," [15].

Walter is new techniques for geo-replica which had properties as storage system for web applications, Walter work on a techniques to avoid conflict occurs in across sites, thereby allowing to implement commit locally in sites, a precisely-stated isolation property that permits asynchronous replication across sites without the need for conflict resolution, A key feature behind Walter is Parallel Snapshot Isolation (PSI), PSI thus permits an efficient, while also providing strong guarantees to applications demonstrated the usefulness of Walter by building a Facebook like application of social networking, and porting a third-party Twitter clone, applications were simple to implement and achieved reasonable performance [16].

A new replication designed to improve consistency of replication in mobile database, the replication strategy consist of three level replication architecture and wheel-based updates propagation protocol working as binary combination to achieve the goal of implementation, the strategy built on supporting frequently disconnected mode, then can synchronize replication in higher level . anew strategy based on a hybrid approach, built to exploit the features of optimistic and pessimistic replication, strategy built on divides data in to frequently changed data and infrequently changed data, update can allowed or facing restricted according to this type , the propagation protocol achieve load balance in propagation and ordering process because, process operation shared by various hosts [17].

New replica propose which is built in idea of using method for recording transactions data for all work done in database source, and forwards the change to the target, which use the method of shadow table and trigger, there is disadvantages of increasing space requirement because there is periodical detection of the table , which is increase process time [18].

Replication strategy, that's has different ways of replicating and managing data on fixed and mobile networks. In the fixed network, the data object is replicated synchronously to all sites in a manner of logical three dimensional grid structure, while in the mobile network, the data object is replicated asynchronously at only one site based on the most frequently visited site. In this strategy, the synchronous replication hinders the fixed network to be scale to wide areas The proposed replication technique is compared with a baseline replication technique and shown to exhibit high availability, fault tolerance and minimal access times of the data and services, which are very important in an environment with low-quality communication links [19].

New replication strategy known as (Cedar) works on preserving eventual consistency by using a Client/Server design; a central server holds the master copy of the database. At infrequent intervals when a client has connection to the server (which may occur hours or days apart), its replica is refreshed from the master copy. Central server is used to hold the master copy in this strategy [20] .

Transaction-Level-Result-Set Propagation is proposed for mobile database replication, each fixed and mobile nodes store a copy replica of data. The mobile node is allowed to update its local replica. However, updates locally committed at the mobile nodes need to be checked at the fixed node before they can be globally committed [21].

\section{REVIEW RESULT}

From the literature review, it is shown that the main approaches used to handle data availability are data replication fig. 5 , and data caching fig. 6 .

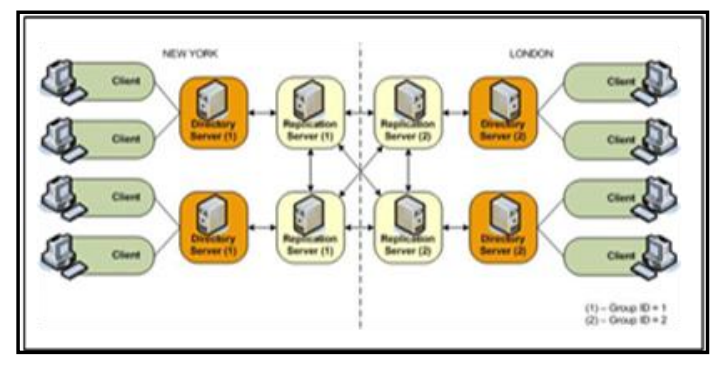

Fig. 5 Data Replication

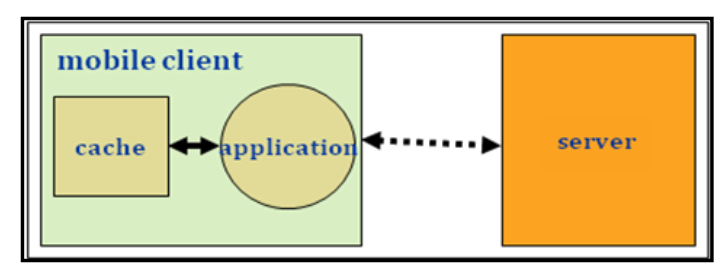

Fig. 6 Data Caching

www.iosrjournals.org 
Previous works in mobile data caching and replication concentrate on how to increase the availability of data by using such ways cooperative and collaborative caching and some of replication methods. But if we trace all this works, mobile unit will not be able to continue working in partial or totally disconnected mode, because some parts of cached data are stored in other places like neighbor nodes. This concept will not support availability of data because mobile unit depends on other locations to answer any query. Also, a node which is having the cached data, or part of it, may not be reachable [19][21][22].

\section{CONCLUSION}

Using mobile devices for conducting business transactions which involves data from a remote location is the issue to be investigated in this study. This study review previous work which is done to support mobile client to proceed with the transactions even during off line or disconnected mode using replication and caching data methods.

\section{REFERENCES}

[1] M. Tarafdar and M. S. Haghjoo, "Location Privacy in Processing Location Dependent Queries in Mobile Database Systems," Computer Engineering, pp. 181-186, 2010

[2] A. A. Fadelelmoula, "Optimistic Replication in Mobile Traffic Control Environment," Information Sciences, pp. 543-548, 2007.

[3] N. Dimokas, D. Katsaros, L. Tassiulas, and Y. Manolopoulos, "High performance, low complexity cooperative caching for wireless sensor networks," Wireless Networks, vol. 17, no. 3, pp. 717-737, Dec. 2010.

[4] Y. Saito, “Optimistic replication,” Science, vol. 37, no. 1, Publisher: ACM Press, Pages: 42-81, 2005.

[5] H. K. Chavan, “A Survey of Mobile Database Cache,” Engineering.

[6] S. K. Madria, "Mobile data management," Ieee Potentials, no. November, pp. 11-15, 2001.

[7] L. Deboosere, B. Vankeirsbilck, P. Simoens, F. D. Turck, B. Dhoedt, and P. Demeester, “Cloud-based Desktop Services for Thin Clients," Ieee Internet Computing, no. ii, pp. 1-6, 2011.

[8] L. Y. and G. Cao, “Supporting Cooperative Caching in Ad Hoc Networks,” IEEE Trans. Mobile Computing, vol. 5, pp. 77-89, 2006.

[9] C. D. S. Lim, W. Lee, G. Cao, "A Novel Caching Scheme for Internet Based Mobile Ad Hoc Networks Performance," Ad Hoc Networks, vol. 4, pp. 225-239, 2006.

[10] K. S. Du, Y., \& Gupta, “COOP: A cooperative caching service in MANETs,”. In Proceedings of ICAS-ICNS, pp. 58-63, 2005.

[11] A. H. Networks, L. Yin, S. Member, and G. Cao, "Supporting cooperative caching in ad hoc networks," IEEE Transactions on Mobile Computing, vol. 5, no. 1, pp. 77-89, Jan. 2006.

[12] G. C. L. Y. C. R. Das, “Cooperative Cache-Based Data Access in Ad Hoc Networks,” ”, IEEE Computer Society, 2004.

[13] N. Chauhan, L. K. Awasthi, and N. Chand, "Data Caching with Intelligent Prefetching in Mobile Ad Hoc Networks," 2011 International Conference on Communication Systems and Network Technologies, pp. 71-75, Jun. 2011.

[14] Z. Itani, “Efficient Pull Based Replication and synchronization for Mobile Databases,” Access, pp. 5-8.

[15] U. F. Jr, R. Pe, F. Cletus, R. P. Valentim, and L. A. Ferreira, “Adaptive Replication Control based on Consensus * Categories and Subject Descriptors," DBMS, pp. 1-10.

[16] Y. Sovran, "Transactional storage for geo-replicated systems," October, pp. 385-400.

[17] A. Ahmed, D. D. Dominic, and A. Abdullah, "A NOVEL REPLICATION STRATEGY FOR LARGE-SCALE MOBILE DISTRIBUTED DATABASE SYSTEMS,” Science And Technology, vol. 6, no. 3, pp. 268 - 299, 2011.

[18] E. F. Khairullah, "Improving Accuracy of Context Aware Data Replication In Mobile Computing Application," Context, pp. 498$503,2009$.

[19] M. D. and M. O. J.Abawajy, “novel data replication and management protocol for mobile computing systems,” Mobile Information Systems, vol. 2, no. Number 1/2006, pp. 3-19, 2006.

[20] N. Tolia, M. Satyanarayanan, and A. Wolbach, "Improving Mobile Database Access Over Wide-Area Networks Without Degrading Consistency," ACM978, pp. 71-84, 2007.

[21] D. Meng Xiaofeng Wang Shan Zhiming, “A transactional asynchronous replication scheme for mobile database systems," Journal of Computer Science and Technology,vol.17,2002. 